\title{
COMMUTATIVE RIMS IN CLANS WITH ZERO
}

\section{EDWARD N. FERGUSON}

A semigroup is a Hausdorff space with a continuous, associative multiplication defined on it. Standard references are [1] and [2]. No notational distinction will be made between the semigroup and the underlying space. If $S$ is a compact semigroup and if $e^{2}=e \in S$, then the (unique) maximal group containing $e$ will be denoted $H(e)$. The (unique) minimal ideal of $S$ will be denoted $M(S)$. A hormos is defined in [1]. For our purposes it is sufficient to know that a hormos is a compact, connected, and abelian semigroup with identity. This paper relies heavily on the Second Fundamental Theorem of [1] and we state the pertinent parts:

Second Fundamental Theorem. Let $S$ be a compact semigroup. Then the following properties are equivalent:

(1) The connected component of each idempotent meets the minimal ideal.

(2) If $e$ is an idempotent, there is an irreducible hormos $T$ in eSe such that $T \cap H(e)=\{e\}$ and $T \cap M(S) \neq \varnothing$.

(3) If $e$ is an idempotent and $A_{e}$ a compact, connected, abelian subgroup of $H(e)$, then there is an irreducible hormos $T$ in the centralizer of $A_{e}$ and in eSe such that $T \cap H(e)=\{e\}$ and $T \cap M(S) \neq \varnothing$.

Throughout this paper cohomology is that of Alexander-Spanier with coefficient group arbitrary.

Definition. Let $X$ be a space and $R$ a compact subset of $X$. Then $R$ is called a rim of $X$ if for each proper closed subset $Y$ containing $R$ there is some integer $n$ such that the map $H^{n}(X) \rightarrow H^{n}(Y)$ induced by inclusion is not onto. (See [3], but note the slight difference.)

LEMma. Let $S$ be a continuum semigroup containing 0 and 1 and let $R$ be a rim of $S$. If $T$ is a continuum subsemigroup containing 0 and 1 , then $S=R T$.

Proof. It is clear that $R \subseteq R T$ and that $R T$ is homotopic to 0 . Hence $H^{n}(S) \rightarrow H^{n}(R T)$ is onto for all $n$. Since $R$ is a rim, $R T=S$. This lemma admits a quick generalization to actions.

THEOREM. Let $S$ be a continuum semigroup with 0 and 1 and let $R$ be a rim of $S$. Assume $R$ is connected and if $x, y \in R$, then $x y=y x$. Then $S$ is commutative.

Presented to the Society, August 28, 1969; received by the editors April 15, 1969. 
Proof. It is known [3] that $1 \in R$. Let $C$ be the smallest compact semigroup in $S$ containing $R$. $C$ is a continuum subsemigroup, $1 \in C$, and $C$ is abelian. Therefore if $e \in M(C)$, then $M(C)=H(e) \cap C$. Also $e S e \cap C=H(e) \cap C$.

By the SFT there is a hormos I in $C$ from 1 to $H(e) \cap C$. There is also a hormos $T$ in $e S e$ from $e$ to 0 and $T \cap H(e)=\{e\}$. We may choose $T$ in the centralizer of $M(C)$ so that $M(C) T$ is a semigroup. Relying on the lemma we have $R(I \cup M(C) T)=S$. Now $R I \subseteq C$ so eSe $\subseteq R M(C) T$. But $R M(C) T \subseteq M(C) T \subseteq H(e) T \subseteq e S e$. Thus eSe $=R M(C) T=M(C) T$ an abelian semigroup.

Now $S=C \cup e S e$ and both members of the union are abelian. Let $c \in C$ and $d \in e S e$. Then $c d=c e d=d c e=d e c e=d e c=d c$. Hence members of $C$ and $e S e$ commute with each other and $S$ is abelian.

Example. Let $S_{1}=\left\{(a, b)\left|(a, b) \in R_{2},\right| a|+| b \mid \leqq 1\right\}$. Define a multiplication on $S_{1}$ by $(a, b)(c, d)=(|a c|,|a d|+|b|) \cdot M\left(S_{1}\right)$ $=\{(0, x) \mid 0 \leqq x \leqq 1\} . S_{1} / M\left(S_{1}\right)$ (the Rees quotient) is a compact semigroup with 0 and connected, commutative rim. However, $S_{1} / M\left(S_{1}\right)$ is not commutative.

Let $S_{2}$ be $[0,1]$ with the usual multiplication.

Take the disjoint union of $S_{1} / M\left(S_{1}\right)$ and $S_{2}$ and identify their respective zeros. If $(a, b) \in S_{1} / M\left(S_{1}\right)$ and $x \in S_{2}$, define

$$
\begin{aligned}
& (a, b) x=(a x, b), \\
& x(a, b)=(a x, b x) .
\end{aligned}
$$

The space $S$ so defined is a continuum semigroup with zero and 1. It has for a rim the rim of $S_{1} / M\left(S_{1}\right)$ together with the identity. The rim is commutative, but $S$ is not.

The author wishes to express his thanks to Professor D. Stadtlander for many helpful conversations.

\section{Bibliography}

1. Karl Heinrich Hofmann and Paul S. Mostert, Elements of compact semigroups, Merrill, Columbus, Ohio, 1966.

2. A. B. Paalman-deMiranda, Topological semigroups, Mathematische Centrum, Amsterdam, 1964.

3. A. D. Wallace, The Gebielstreue in semigroups, Indag. Math. 18 (1956), 271-274.

UNIVERSITY OF FLORIDA 